\title{
Patterns of plant use in religious offerings in Bali (Indonesia)
}

\author{
Wawan Sujarwo ${ }^{1 *}$, Giulia Caneva ${ }^{2}$ and Vincenzo Zuccarello ${ }^{3}$ (1)
}

Received: March 30, 2019

Accepted: August 23, 2019

\begin{abstract}
Balinese Hinduism has incorporated local animistic traditions and offerings, which play a key role in the religious ceremonies called "five holy ceremonies" or Panca yadnya. Since plants constitute fundamental elements of these offerings, we aimed to contribute to their knowledge. We analyzed plants used during ceremonies by interviewing key informants in four ancient villages of Bali (Bali aga). We identified exclusive and common species associated with different kinds of ceremonies and assessed whether there was any pattern in the selection of plants for the various offerings. We recorded 125 species (112 genera, 49 families), most of which belong to the wild ethnoflora of Bali, but also 36 species that are not native to the Malesian region. The religious relationships among ceremonies, called yadnyas, reveal specific compositions of the offerings, with the plants falling into two main groups: common, which comprises 58 plants shared by all yadnyas, and specific, mostly connected to a single yadnya. This pattern of plant use is similar to the previously detected pattern in the traditional ecological knowledge (TEK) of aga villages. The use of plants for Panca yadnya can help avoid cultural erosion related to globalization.
\end{abstract}

Keywords: Bali aga, ceremonies and rituals, floristic similarity, orthopraxy, plant pool, traditional ecological knowledge

\section{Introduction}

Hinduism gives great importance to plants and, in particular, trees and forests (Krishna 2017). A fundamental sense of harmony with nature is shared in several Hindu texts (Hockings 1993; Jansen 1993; Jones \& Ryan 2007; Cush et al. 2008), where forests are depicted as a sources of life and inspiration. As a matter of fact, some trees are worshipped and associated with deities, and have become part of the Hinduism mythology (Sharma 2003; Krishna 2017).

Balinese Hinduism has diverged from the Indian Hinduism by absorbing local practices of local animistic indigenous religions (Jones \& Ryan 2007). For instance, Balinese people often use plants or plant parts to make offerings to ancestors, spirits, and supernatural forces
(Belo 1960; Geertz \& Geertz 1975; Geertz 1980). In Bali, a set of Hinduism principles is represented in a complex ancestral cult, with gods and devils but also deities of fertility, fire, water, the earth, the sun, mountains and the sea (Covarrubias 1937). Balinese Hindus do not recognize a secular reality because their philosophy is based on an understanding that the world in its materialistic aspects is determined by the invisible power of spiritual beings (Picard 2011).

In Bali, traditional and religious ceremonies are more frequent than in any other place in the wider Hindu world (Jones \& Ryan 2007). Offerings are a key element in these ceremonies, and some are quite elaborate, making them one of the most stunning local cultural phenomena, highly appreciated by tourists (Barth 1993; Hobart et al. 2001; Bakan 2011). Balinese Hindus use the term yadnya to describe the ritual ceremonies involving offerings (often

1 Bali Botanical Gardens, Indonesian Institute of Sciences, 82191, Bali, Indonesia

2 Department of Science, University Roma Tre, 00146, Rome, Italy

3 Department of Sciences and Biological and Environmental Technology, Salento University, 73100, Lecce, Italy

* Corresponding author: wawan.sujarwo@lipi.go.id 
material, such as plants, animals, metals) (Monier-Williams et al. 1992; Barth 1993). There are five kinds of yadnyas in Balinese Hinduism, related to different forms of worship, and they are collectively called Panca yadnya (panca meaning five). Plants, or their parts, are the most important element in material offerings and probably provide symbolic meaning related to the ceremonies. Plant species used in Balinese Hinduism offerings were not deeply analyzed, and we wish to test if their patterns of uses are characteristics of the ceremonies, and if the choice of plants can be influenced by different ceremonial meaning.

\section{Balinese Hinduism ceremonies}

What mostly surprises tourists in Bali about the Balinese Hinduism is the great deal of ceremonies and rituals, their attractive aspects, the fervent devotion and the active involvement of local people. Geertz (1973) even said that the Balinese people seem more busy practicing than believing in their religion and many anthropologists agree with this idea (Yamashita 2003; Picard 2011). For instance, Acri (2011) says that "more attention is given to a correct conduct, ethical and liturgical, (orthopraxy) rather than a right belief (orthodoxy)", and the approach of Balinese towards religion and spirituality is more practical (related practices that need to be carried out) than doctrinal. Even though the Balinese Hinduism has ancient Indian roots - religious teachings have been based on oral tradition or traditional performances about stories from Indian epic poems - there has not been a holy Book of Balinese Hinduism until the mid-20th century (Dibia \& Ballinger 2011). As such, traditional ceremonies have a great relevance to the transmission of religion in Bali, and they are a crucial element to understanding the Balinese Hinduism (Hornbacher 2011).

Balinese people believe in a Supreme God manifested in three main forms: i.e., the Creator, the Preserver, and the Destroyer (more worshipped than the other two) (Wiener 1995). All deities are worshipped through daily and periodic offerings, with celebrations and events in villages and temples (Jones \& Ryan 2007). A general description of the five groups of ceremonies (Putra 1988) is provided: 1) Bhuta yadnya is a ceremony of offerings to spirits and demons, i.e. Pengerupukan by burning a giant puppet (ogohogoh) the day before Silent Day (Nyepi). 2) Dewa yadnya is a set of rituals to worship gods and deities, i.e., the recurrent ceremonies of the full moon and new moon, the annual Silence Day, and several bi-annual ceremonies including Galungan and Kuningan (both are relevant feasts for Hindus in Bali; Galungan refers to the time when the ancestral spirits visit the Earth. The last day of the celebration is Kuningan, when they return). 3) Manusa yadnya is intended to celebrate the different stages of human life. There are 13 ceremonies in Manusa yadnya that use plants as symbols. They include the tooth filing ceremony - where the upper front teeth are filed flat - and are performed to rid the spirit of the six negative emotions in humans (lust, greed, anger, confusion, drunkenness and jealousy). 4) Pitra yadnya is a ceremony for death and reincarnation. This ceremony aims to restore the body and soul to their place of origin through burial or cremation (Ngaben). Various plants are used during this ritual. 5) Rsi yadnya is a consecration of the clergy and it is carried out with the nomination of a new priest. Reed leaves are often tied around the head of the celebrated priest.

\section{Materials and methods}

\section{Study area}

The island of Bali has a land surface of 5,577 km2, with less than $20 \%$ covered by forests $(7.8 \%$ primary forests, $10.1 \%$ secondary forests, and $0.3 \%$ artificial forests (BPS 2017). The study was conducted in four Bali aga villages located in the northern part of the island (Fig. 1). The people of these four villages belong to the Bali aga ethnic group. Bali aga people are considered as the native Balinese, since their ancestors have lived on the island long before the 15th century when the later Bali people (known as Bali Majapahit) firstly arrived on the island (Sujarwo et al. 2015). Bali aga people have maintained a traditional lifestyle including ancient Hindu traditions and an economy mostly based on agriculture (e.g., green vegetables, fruits, beans, and rice) (Sujarwo \& Caneva 2015). A detailed analysis of the factors (e.g., age, gender, education level) affecting differences in traditional knowledge of plant uses in the surveyed villages is provided in Sujarwo et al. (2014). Following our previous work (Caneva et al. 2017), we decided to select these four aga villages for the present study, since they represent the core of the Traditional Ecological Knowledge (TEK) of the aga ethnicity.

\section{Data collection}

We carried out key informant interviews, following general guidelines for conducting ethnobotanical studies (Alexiades \& Sheldon 1996), to obtain data regarding the plants used in offerings of the Panca yadnya. We used a snowball method to select key informants (Bernard 2002) because it resulted to be the best option for our surveys according to previous experiences in the area (Sujarwo et al. 2014; Caneva et al. 2017). We sought information about potential key informants from village leaders and/ or religious leaders, and then the first key informants led us to the next key informants in their village.

Before each interview, prior informed consent (Rosenthal 2006) was requested and during the interview process we followed international codes of ethics (ISE 2006). After obtaining consent, we were able to speak with twenty informants (five informants in each village, 10 males and 
10 females) in December 2017. Informants' ages spanned from 42 to 81 years. Male informants were mostly Balinese Hindu Priests, and teachers (in their role as guru). The female informants were comprised mostly of tukang banten, specialized craftswomen with skills in producing elements of religious offerings (Barth 1993). Interviews were conducted by the first author in the Balinese language.

We then asked informants to list all the plants (wild and semi-wild or cultivated) that they use or have used as materials of offerings for religious ceremonies, and then we asked details about the plants and their uses (i.e., the name of the plant, the parts used, and which type of yadnya were such parts of the plant used). Wild plants refers to the species grown or produced without cultivation or human care, and semi-wild refers to partially managed plants (Menendez-Baceta et al. 2012).Plant specimens were collected with the informants, prepared as herbarium specimens (Martin 2004), and then identified and deposited at Herbarium Hortus Botanicus Baliense (THBB) in the Bali Botanical Gardens. Some common plant species were directly identified in the field. The scientific nomenclature used in this study has been verified using online sources (i.e., The Plantlist 2018) and the floristic regions of the plant species were obtained from Takhtajan (1986).

\section{Data analysis}

We performed quantitative analyses based on a presence/ absence data matrix $\mathbf{S}$ of plant parts used in the Panca yadnya (148 plant parts X 5 yadnyas) in order to obtain different pools of vegetal materials used in offerings and to assess similarities among the different types of yadnyas.

\section{Exclusive plant part pools}

We determined the exclusive plant part pools using a combinatorial approach (Loehr 2017) based on the matrix $\mathbf{S}$ and on the set of plant parts, P. Starting from the set of

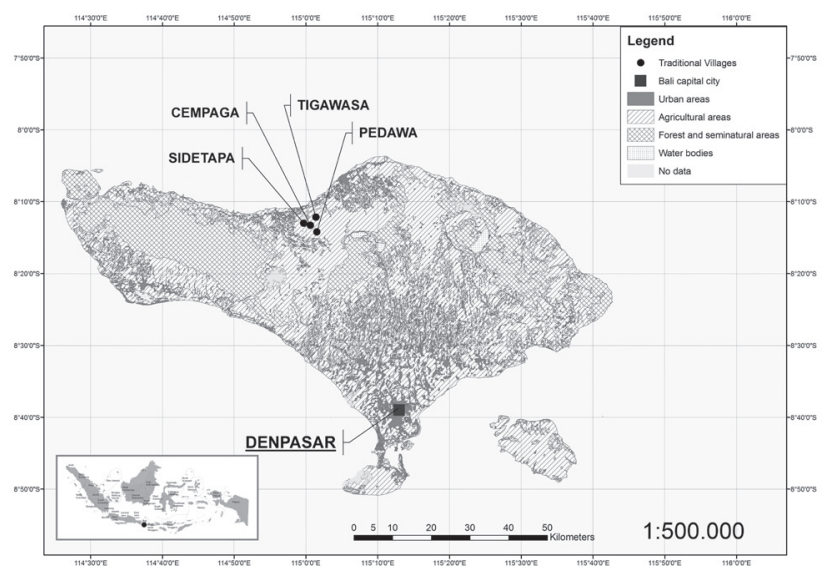

Figure 1. Study area. all combinations of yadnyas, $\mathbf{C}$, given by the power set of the set of yadnyas, $\mathbf{Y}$, all possible 'not empty' pools of plant parts were defined according the following property:

Pool (ci) $=$ \{plant partj $\mid$ plant partj is only present in yadnya of ci combination $\}, \mathrm{i}=1,25, \mathrm{j}=1,148$. Where $\mathrm{ci}=$ the

Table 1. General floristic diversity in Panca yadnya.

\begin{tabular}{|c|c|c|}
\hline \multicolumn{3}{|c|}{ A) General Diversity } \\
\hline & & Element number \\
\hline \multicolumn{2}{|c|}{ Plant parts } & 148 \\
\hline \multicolumn{2}{|c|}{ Species } & 125 \\
\hline \multicolumn{2}{|c|}{ Genera } & 112 \\
\hline \multicolumn{2}{|c|}{ Family } & 49 \\
\hline \multicolumn{2}{|c|}{ Poaceae } & 15 \\
\hline \multicolumn{2}{|c|}{ Leguminosae } & 12 \\
\hline \multicolumn{2}{|c|}{ Zingiberaceae } & 8 \\
\hline \multicolumn{2}{|c|}{ Arecaceae } & 6 \\
\hline \multicolumn{2}{|c|}{ Phyllantaceae } & 5 \\
\hline \multicolumn{2}{|c|}{ Apocynaceae } & 5 \\
\hline \multicolumn{2}{|c|}{ Type life form } & Species \\
\hline \multicolumn{2}{|c|}{ Tree } & 48 \\
\hline \multicolumn{2}{|c|}{ Herb } & 31 \\
\hline \multicolumn{2}{|c|}{ Shrub } & 28 \\
\hline \multicolumn{2}{|c|}{ Climber } & 18 \\
\hline \multicolumn{2}{|c|}{ Wild and Semi-wild } & 67 \\
\hline \multicolumn{2}{|c|}{ Cultivated } & 63 \\
\hline \multicolumn{2}{|c|}{ Both } & 5 \\
\hline \multicolumn{3}{|c|}{ B) Diversity in yadnya } \\
\hline Types of offerings & Species & Plant part \\
\hline Pitra yadnya & 118 & 136 \\
\hline Bhuta yadnya & 76 & 86 \\
\hline Dewa yadnya & 70 & 76 \\
\hline Manusa yadnya & 67 & 74 \\
\hline Rsi yadnya & 57 & 60 \\
\hline \multicolumn{3}{|c|}{ Used parts } \\
\hline \multicolumn{2}{|c|}{ Leaves } & 52 \\
\hline \multicolumn{2}{|c|}{ Fruits } & 33 \\
\hline & wers & 16 \\
\hline & eds & 11 \\
\hline & ber & 11 \\
\hline & od & 10 \\
\hline & $\operatorname{lm}$ & 6 \\
\hline & em & 3 \\
\hline & ark & 2 \\
\hline & ap & 1 \\
\hline & fibres & 1 \\
\hline & drib & 1 \\
\hline & e part & 1 \\
\hline & C) Habitat and $\mathrm{Ve}$ & \\
\hline Habitat types & \begin{tabular}{|l} 
Species \\
\end{tabular} & $\%$ \\
\hline Tropical & 115 & 77.18 \\
\hline Subtropical & 26 & 17.45 \\
\hline Temperate & 8 & 5.37 \\
\hline & Vegetation ty & \\
\hline Forests & 135 & 90.6 \\
\hline Grasslands & 13 & 8.72 \\
\hline Ponds/Lakes & 1 & 0.67 \\
\hline
\end{tabular}


i-th element of $\mathbf{C}$; plant partj = the j-th element of $\mathbf{P}$; Pool (ci) = the set of plant parts associated to the ci combination.

According to this criterion, a plant part belongs to a specific pool if it is only utilized in the yadnya present in the combination related to such pool. Each pool of plant parts is defined and described by the yadnya in which it is used exclusively

\section{Similarities in yadnya}

We used the resemblance function based on the Jaccard coefficient (Jaccard 1901) to calculate pair-wise similarities between yadnyas in the data matrix $\mathbf{S}$. The average similarity of every yadnya was used to assess a global gradient of similarity in the dataset.

\section{Results}

Plant offerings can be seen everywhere, especially the Canangsari (Fig. 2), a tiny coconut leaf basket filled with rice, fruit, and flowers, often in front of houses, shops, hotels and even on cars and motorbikes. There are more elaborate offerings in shrines and temples, and even large offerings, such as the penjor, a three-meter bamboo culm with many elements attached for decoration used in special ceremonies (i.e., galungan feast celebration) (Eiseman 1990).

\section{General floristic diversity of ethnoflora of offerings in Panca yadnya}

We noted the use of 125 species of plants (including 148 plant parts) from 112 genera and 49 families that are used in rituals of offerings in Bali. There are 67 wild and semiwild species, 63 cultivated species, and five species are both wild and cultivated (Supplementary material). Six families were considered particularly important in the Panca yadnya by the local inhabitants: Poaceae (15 species), Fabaceae (12 species), Zingiberaceae (eight species), Arecaceae (six species), Phyllantaceae (five species), and Apocynaceae (five species). The dominant life forms are trees, followed by herbs, shrubs, and climbers. The number of plant species used in the Panca yadnya is quite variable: Pitra yadnya with 118 species (136 plant parts), followed by Bhuta yadnya, Dewa yadnya, Manusa yadnya, and Rsi yadnya (Fig. 3). The most frequently used parts are the leaves followed by fruits and other plant parts (Tab. 1). Most plant parts are collected throughout the year.

The 125 recorded species include tropical plants (77\%), subtropical plants (18\%), and temperate plants (5\%), of which $71.2 \%$ are native to the Malesian floristic region, $38.4 \%$ to the Indochinese floristic region, and $34.4 \%$ to the Indian floristic region. The considerable percentage of species of the Malesian region is possibly influenced by cultural influences. The study recorded 36 species that are not native to the Malesian region (Fig. 4).

\section{Species pools in Panca yadnya}

Table 2 describes pools of species, or its parts, exclusively present in a specific yadnya combination. Among all possible combinations (32), less than $50 \%$ were not empty combinations (14). The two most frequently occurring species show a bimodal distribution, representing opposite conditions - a combination with only one yadnya and all yadnyas, i.e., two maxima related respectively to a common pool (58) and to exclusive pools of a single yadnya (Fig. 5). Only a single plant part (the flowers of Canaga odorata) is exclusively used in four yadnyas. Also, leaves of Arenga pinnata are used in offerings prepared in all yadnya, while its black fibers, fruits, and sap represent exclusive elements of, respectively, Dewa, Manusa, and Butha yadnyas (Fig. 6).

\section{Floristic similarity in Panca yadnya}

The similarities between yadnyas, obtained with the Jaccard coefficient, are shown in Figure 7, where yadnyas are ordered according to the increasing values of total richness of plant parts. The average similarity for a single yadnya ranges from $0.5-0.67$ and there is a rather high variability of resemblances between yadnyas based on floristic data, with the highest value observed between Manusa yadnya and Rsi yadnya (0.81), while the lowest one was between Pitra yadnya and Rsi yadnya (0.43). In Figure 7, it is notable the separation of Pitra yadnya and, to a lesser extent, of Bhuta yadnya from the others.

\section{Discussion}

\section{Ethnoflora of offerings in Panca yadnya}

An offering can be seen as a sort of self-sacrifice, as people spend time and money to make objects to offer (Eiseman 1990). In Bali, offerings are often labor-intensive as they should be attractive, not necessarily complex (although often so), and well prepared when presented to higher aspects of God. However, if they are offered to negative forces and demons, they may be less carefully composed. Also, with minor exceptions regarding the worship of demons, an offering must be fresh and cannot be used more than once. Though beautiful, offerings are never long-lasting because they are mainly made of natural materials directly coming from Balinese customs and traditions (Eiseman 1990) and plants are a key element both for their appearance and symbolic meaning.

Species, or their parts, are used to convey general religious meanings in the various ceremonies and it can be inferred their relationship with right practices of custom and rituals. From our results, the high diversity in species of the Balinese ethnoflora of offerings seems to be correlated to the heterogeneity of religious customs (Reuter 2012), and 


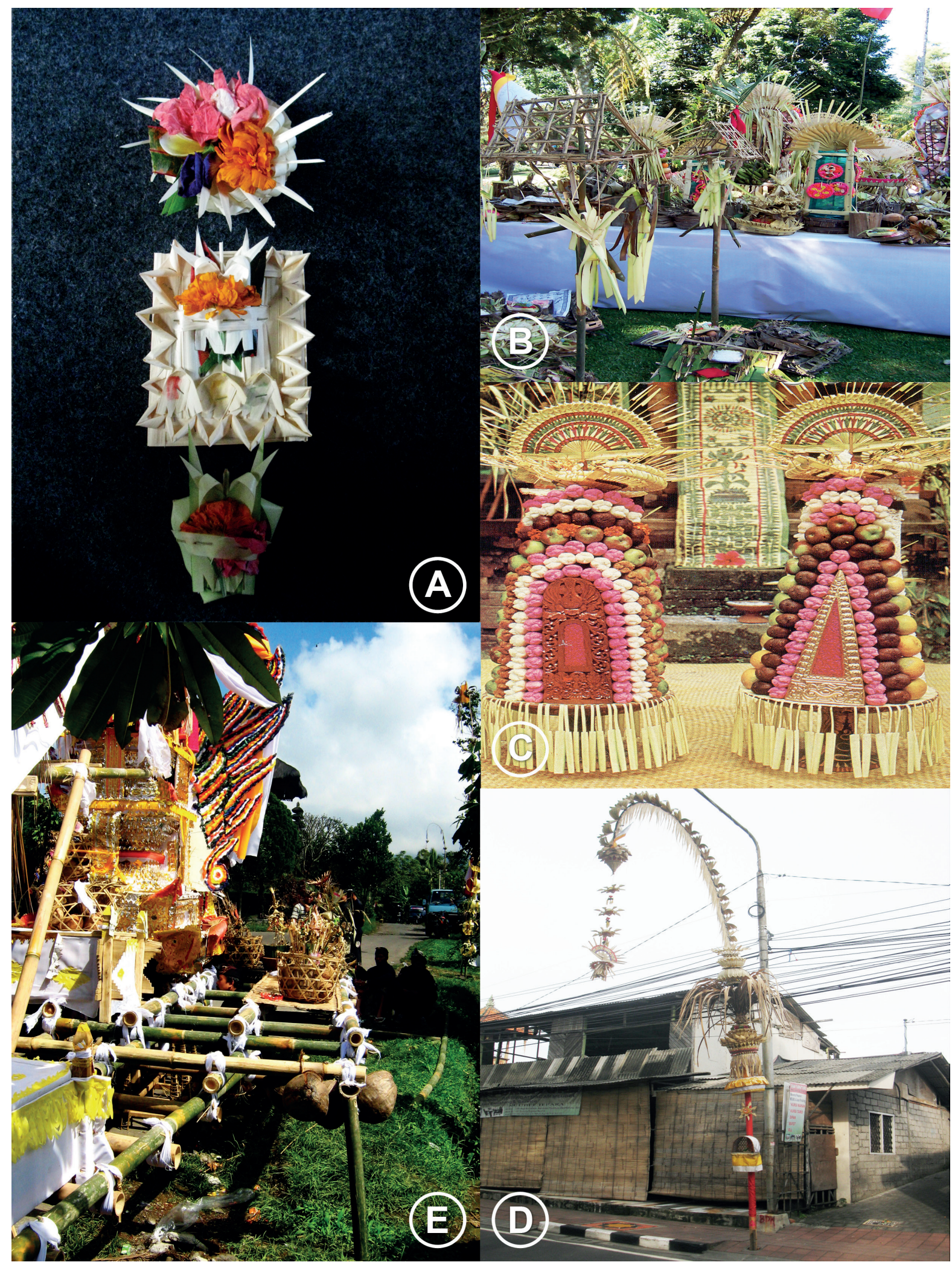

Figure 2. Example of offerings in Balinese Hinduism (A. Canangsari; B. Mecaru; C. Banten; D. Penjor; E. Ngaben). Notes: Canangsari is one of the daily offerings; Mecaru is one of the Butha kala ceremonies that aim to keep balance between the macrocosm (universe) and microcosm (our inner world); Banten is an offering for gods/spirits and encapsulates Bali's unique fusion of Hinduism; Penjor is one of the offerings used by Balinese Hindus as part of most important ceremony, especially for the anniversary of temples and Galungan celebrations (one of the biggest feasts for Balinese Hindus); Cremation (Ngaben) is the common word used for the Pitra yadnya ceremony. 


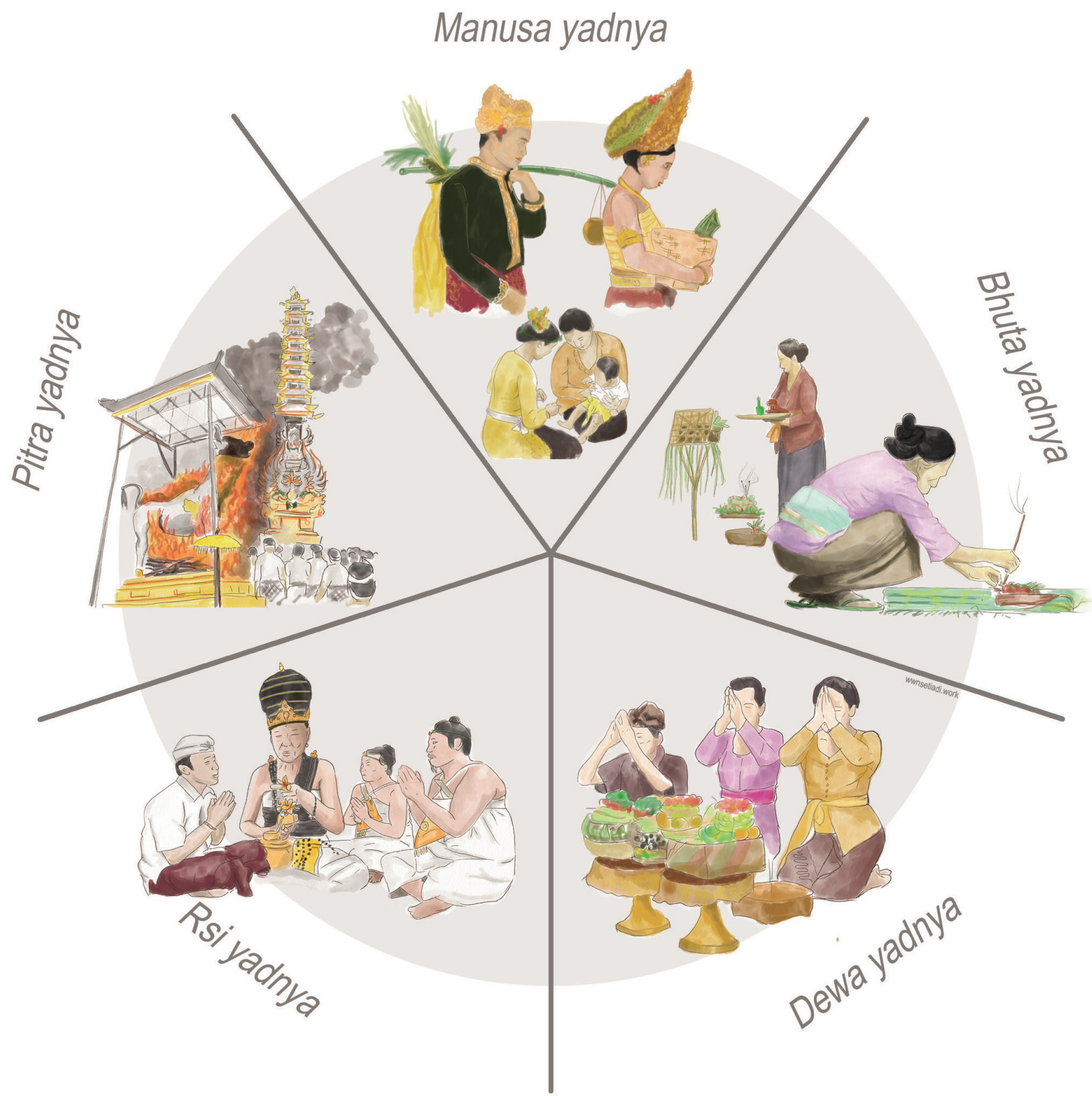

Figure 3. The main five Balinese Hinduism ceremonies (Panca yadnya).

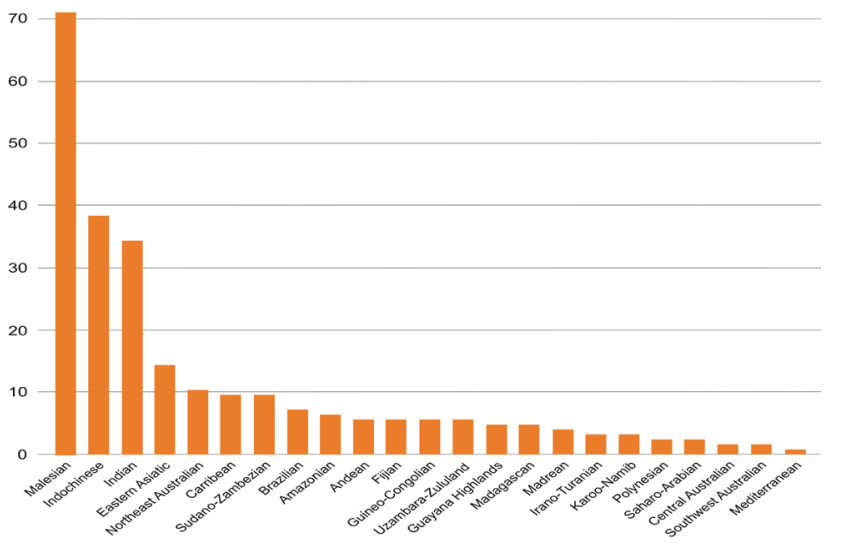

Figure 4. Occurrence of the ceremonial species from the different Floristic Regions.

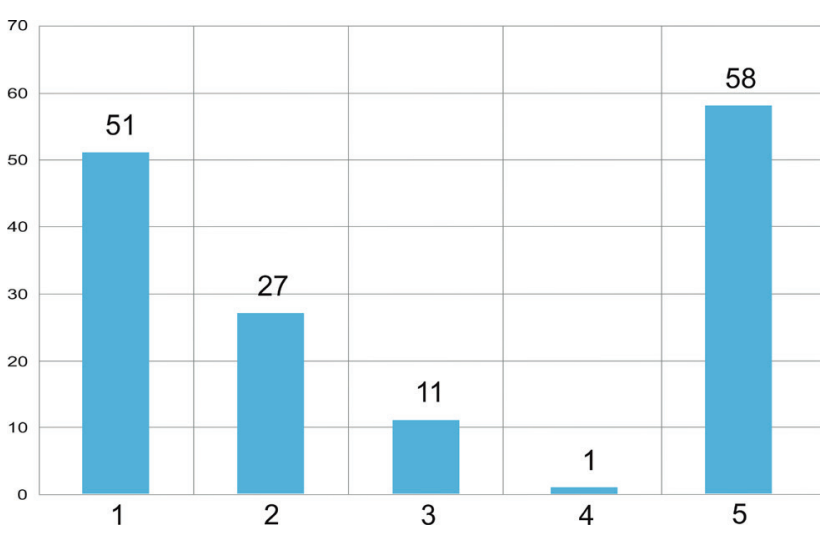

Figure 5. Plant parts exclusively present in different numbers of yadnya. 


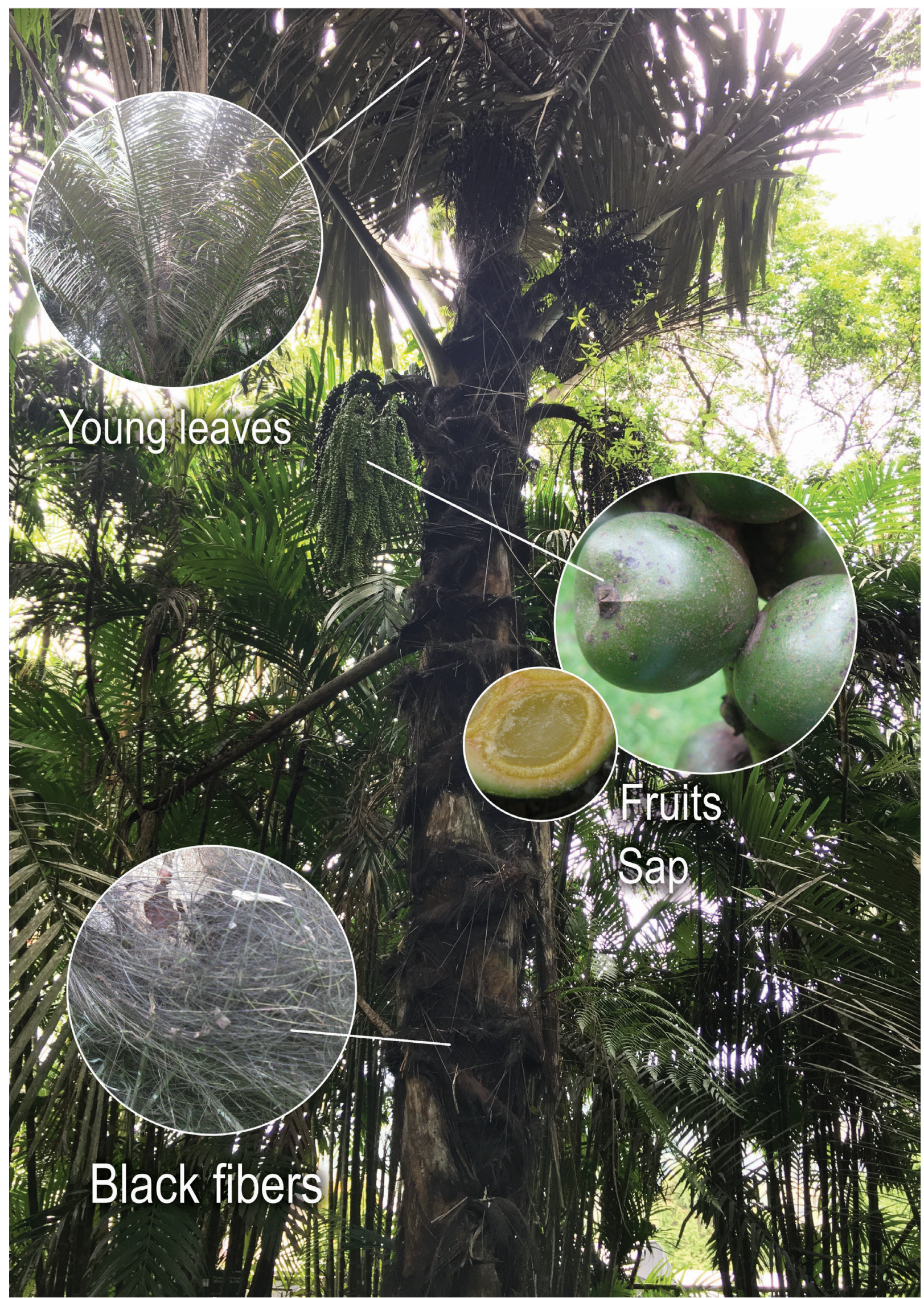

Figure 6. Arenga pinnata, an example of one of the most-used plant species in Panca yadnya. 


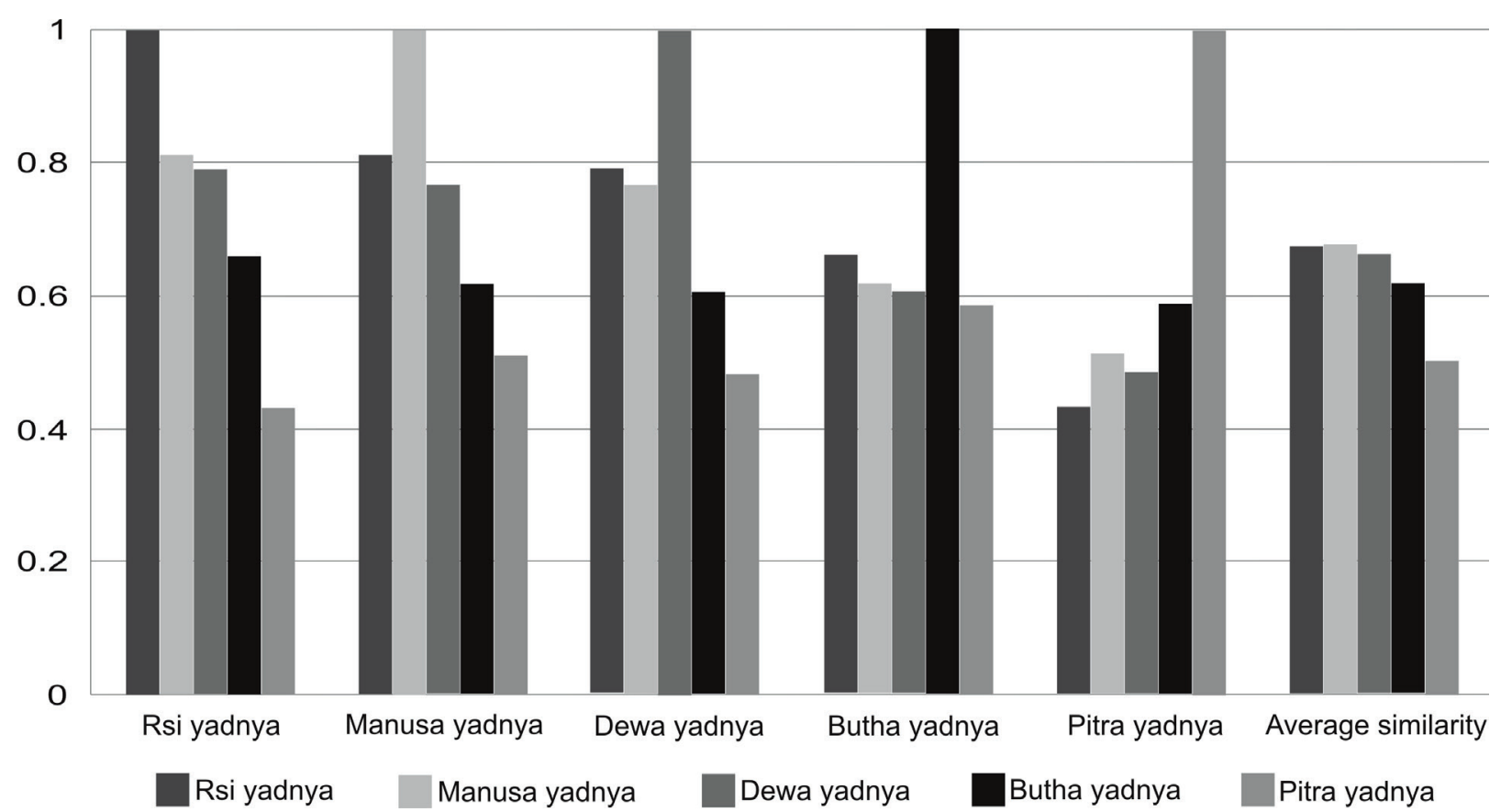

Figure 7. Similarities between yadnya based on used plant parts.

Table 2. Subsets of plant species, with indication of their use in the different kinds of yadnyas.

\begin{tabular}{|c|c|c|c|c|c|c|c|c|c|c|c|}
\hline \multirow{2}{*}{ No. } & \multirow{2}{*}{$\begin{array}{l}\text { Plant families \& species, } \\
\text { [voucher specimen code] }\end{array}$} & \multirow[b]{2}{*}{ Life form } & \multirow[b]{2}{*}{ Vernacular names } & \multirow[b]{2}{*}{$\begin{array}{l}\text { Used } \\
\text { parts }\end{array}$} & \multicolumn{5}{|c|}{ Use in yadnya } & \multirow[b]{2}{*}{ Villages } & \multirow[b]{2}{*}{$\begin{array}{l}\text { Number of } \\
\text { Informants }\end{array}$} \\
\hline & & & & & $\begin{array}{l}\text { Dewa } \\
\text { yadnya }\end{array}$ & $\begin{array}{c}\text { Pitra } \\
\text { yadnya }\end{array}$ & $\begin{array}{c}\text { Manusa } \\
\text { yadnya }\end{array}$ & $\begin{array}{l}\text { Bhuta } \\
\text { yadnya }\end{array}$ & $\begin{array}{c}\text { Rsi } \\
\text { yadnya }\end{array}$ & & \\
\hline & Acanthaceae & & & & & & & & & & \\
\hline 1 & $\begin{array}{c}\text { Asystasia mysorensis (Roth) T. Anderson } \\
\text { [WS200] }\end{array}$ & Herb & Knuja & Leaves & & 1 & & & & $\mathrm{P}$ & 2 \\
\hline 2 & Barleria prionitis L. [WS201] & Herb & Landep-landep & Leaves & 1 & & & & & C & 2 \\
\hline 3 & Graptophyllum pictum (L.) Griff. [WS202] & Herb & Temen & Leaves & 1 & 1 & 1 & 1 & 1 & C, S & 6 \\
\hline \multirow[t]{2}{*}{4} & Justicia gendarussa Burm.f. [WS203] & Shrub & Dusakiling & Leaves & 1 & 1 & 1 & 1 & 1 & C, P & 4 \\
\hline & Achariaceae & & & & & & & & & & \\
\hline \multirow[t]{2}{*}{5} & Pangium edule Reinw. [WS204] & Tree & Pangi & Fruit & 1 & 1 & 1 & 1 & 1 & C, P, S & 9 \\
\hline & Amaranthaceae & & & & & & & & & & \\
\hline 6 & Celosia spicata Spreng. [WS205] & Shrub & Keniwan & Flower & & 1 & & & & $\mathrm{P}$ & 1 \\
\hline \multirow[t]{2}{*}{7} & Gomphrena globosa L. [WS206] & Shrub & Ratna & Flower & 1 & 1 & 1 & & & $\begin{array}{l}\text { C, P, } \\
\text { S, T }\end{array}$ & 15 \\
\hline & Amaryllidaceae & & & & & & & & & & \\
\hline 8 & Allium cepa L. [WS207] & Herb & Bawang merah & Tuber & 1 & 1 & 1 & 1 & 1 & $\begin{array}{l}\text { C, P, } \\
\text { S, T }\end{array}$ & 8 \\
\hline \multirow[t]{2}{*}{9} & Allium sativum L. [WS208] & Herb & $\begin{array}{l}\text { Bawang putih/ } \\
\text { kesuna }\end{array}$ & Tuber & 1 & 1 & 1 & 1 & 1 & $\begin{array}{l}\text { C, P, } \\
\text { S, T }\end{array}$ & 10 \\
\hline & Anacardiaceae & & & & & & & & & & \\
\hline \multirow[t]{2}{*}{10} & Mangifera caesia Jack [WS 209] & Tree & Wani/poh & Fruit & 1 & 1 & 1 & 1 & 1 & $\mathrm{P}, \mathrm{S}, \mathrm{T}$ & 7 \\
\hline & Annonaceae & & & & & & & & & & \\
\hline \multirow[t]{2}{*}{11} & $\begin{array}{c}\text { Cananga odorata (Lam.) Hook.f. \& Thomson } \\
\text { [WS210] }\end{array}$ & Tree & Sandat & Flower & 1 & 1 & 1 & & 1 & $\begin{array}{l}\text { C, P, } \\
\text { S, T }\end{array}$ & 17 \\
\hline & Apocynaceae & & & & & & & & & & \\
\hline 12 & Hoya heuschkeliana Kloppenb. [WS211] & Climber & Tebel-tebel & Leaves & 1 & & & & & C & 2 \\
\hline 13 & Plumeria alba L. [WS212] & Tree & Jepun & Flower & 1 & 1 & 1 & 1 & 1 & $\begin{array}{l}\text { C, P, } \\
\text { S, T }\end{array}$ & 20 \\
\hline 14 & Alstonia scholaris (L.) R. Br. [WS213] & Tree & $\begin{array}{l}\text { Polegamongan/ } \\
\text { pulai }\end{array}$ & Leaves & & 1 & & 1 & & C & 2 \\
\hline 15 & Calotropis gigantea (L.) Dryand. [WS214] & Shrub & Medori putih & Flower & & 1 & & & & $\mathrm{~T}$ & 4 \\
\hline 16 & Nerium oleander L. [WS215] & Shrub & Kenyeri putih & Flower & & 1 & 1 & & & $\mathrm{~T}$ & 5 \\
\hline \multirow[t]{2}{*}{17} & $\begin{array}{l}\text { Tabernaemontana divaricata (L.) R.Br. ex Roem. } \\
\text { \& Schult. [WS216] }\end{array}$ & Shrub & Tuludnyuh & Flower & & 1 & & & & C & 2 \\
\hline & Araceae & & & & & & & & & & \\
\hline 18 & Colocasia esculenta (L.) Schott [WS217] & Shrub & $\begin{array}{c}\text { Keladi/don } \\
\text { kembang }\end{array}$ & Tuber & 1 & 1 & 1 & & & $\begin{array}{l}\text { C, P, } \\
\text { S, T }\end{array}$ & 16 \\
\hline
\end{tabular}


Table 2. Cont.

\begin{tabular}{|c|c|c|c|c|c|c|c|c|c|c|c|}
\hline \multirow[b]{2}{*}{ No. } & \multirow[b]{2}{*}{$\begin{array}{l}\text { Plant families \& species, } \\
\text { [voucher specimen code] }\end{array}$} & \multirow[b]{2}{*}{ Life form } & \multirow[b]{2}{*}{ Vernacular names } & \multirow[b]{2}{*}{$\begin{array}{l}\text { Used } \\
\text { parts }\end{array}$} & \multicolumn{5}{|c|}{ Use in yadnya } & \multirow[b]{2}{*}{ Villages } & \multirow[b]{2}{*}{$\begin{array}{l}\text { Number of } \\
\text { Informants }\end{array}$} \\
\hline & & & & & $\begin{array}{c}\text { Dewa } \\
\text { yadnya }\end{array}$ & \begin{tabular}{|c|} 
Pitra \\
yadnya
\end{tabular} & $\begin{array}{l}\text { Manusa } \\
\text { yadnya }\end{array}$ & $\begin{array}{c}\text { Bhuta } \\
\text { yadnya }\end{array}$ & $\begin{array}{c}\text { Rsi } \\
\text { yadnya }\end{array}$ & & \\
\hline \multirow{3}{*}{19} & Araliaceae & & & & & & & & & & \\
\hline & Schefflera elliptica (Blume) Harms [WS218] & Climber & Tulak & Wood & & 1 & & 1 & & $\begin{array}{l}\text { C, P, } \\
\text { S, T }\end{array}$ & 15 \\
\hline & Arecaceae & & & & & & & & & & \\
\hline 20 & Areca catechu L. [WS219] & Tree & Pinang & Fruit & 1 & 1 & 1 & 1 & 1 & $\begin{array}{l}\text { C, P, } \\
\text { S, T }\end{array}$ & 20 \\
\hline \multirow{4}{*}{21} & \multirow{4}{*}{ Arenga pinnata (Wurmb) Merr. [WS220] } & \multirow{4}{*}{ Tree } & \multirow{4}{*}{$\begin{array}{c}\text { Aren/jaka/ } \\
\text { beluluk/enau }\end{array}$} & Leaves & 1 & 1 & 1 & 1 & 1 & $\begin{array}{l}\text { C, P, } \\
\text { S, T }\end{array}$ & 20 \\
\hline & & & & Fruit & & & 1 & & & $\begin{array}{l}\text { C, P, } \\
\text { S, T }\end{array}$ & 20 \\
\hline & & & & $\begin{array}{l}\text { Black } \\
\text { fibres }\end{array}$ & 1 & & & & & $\begin{array}{l}\text { C, P, } \\
\text { S, T }\end{array}$ & 20 \\
\hline & & & & Sap & & & & 1 & & S & 1 \\
\hline 22 & Caryota mitis Lour. [WS221] & Tree & Dudu & Stem & 1 & 1 & 1 & 1 & 1 & C, S, T & 11 \\
\hline \multirow{3}{*}{23} & \multirow{3}{*}{ Cocos nucifera L. [WS222] } & \multirow{3}{*}{ Tree } & \multirow{3}{*}{$\begin{array}{c}\text { Kelapa/nyuh } \\
\text { gading/nyuh } \\
\text { gadang/nyuh } \\
\text { sudamala }\end{array}$} & Fruit & 1 & 1 & 1 & 1 & 1 & $\begin{array}{l}\text { C, P, } \\
\text { S, T }\end{array}$ & 20 \\
\hline & & & & Leaves & 1 & 1 & 1 & 1 & 1 & $\begin{array}{l}\text { C, P, } \\
S, T\end{array}$ & 20 \\
\hline & & & & Midrib & & & & 1 & & S & 2 \\
\hline 24 & $\begin{array}{l}\text { Pinanga coronata (Blume ex Mart.) Blume } \\
\text { [WS223] }\end{array}$ & Tree & Peji & Stem & 1 & 1 & 1 & 1 & 1 & C, S, T & 12 \\
\hline \multirow[t]{3}{*}{25} & \multirow[t]{2}{*}{ Salacca zalacca (Gaertn.) Voss [WS224] } & Shrub & Salak & Fruit & 1 & 1 & 1 & 1 & 1 & $\begin{array}{l}\mathrm{C}, \mathrm{P} \\
\mathrm{S}, \mathrm{T}\end{array}$ & 10 \\
\hline & & & & Leaves & & 1 & & & & C, S & 4 \\
\hline & Asparagaceae & & & & & & & & & & \\
\hline 26 & Cordyline fruticosa (L.) A.Chev. [WS225] & Shrub & Andong bang & Leaves & & 1 & & 1 & & $\begin{array}{l}\text { C, P, } \\
\text { S, T }\end{array}$ & 10 \\
\hline 27 & Dracaena angustifolia (Medik.) Roxb. [WS226] & Shrub & Kayu sugih & Leaves & 1 & 1 & 1 & 1 & 1 & S & 4 \\
\hline & Athyriaceae & & & & & & & & & & \\
\hline 28 & Diplazium esculentum (Retz.) Sw. [WS227] & Herb & Paku jukut & Leaves & 1 & 1 & 1 & & & $C, S$ & 4 \\
\hline & Burseraceae & & & & & & & & & & \\
\hline 29 & Pratium iavanicum Burm f [WS228] & Tree & Tenogulun & Wood & & 1 & & 1 & & $S$ & 1 \\
\hline 29 & Prottum javanicum Burm.I. [WSLZ8] & Iree & Ienggulun & Leaves & & 1 & & 1 & & $S$ & 2 \\
\hline & Caricaceae & & & & & & & & & & \\
\hline 30 & Carica papaya L. [WS229] & Tree & $\begin{array}{l}\text { Gedang/ } \\
\text { pepaya }\end{array}$ & Fruit & & 1 & & 1 & & C & 3 \\
\hline & Clusiaceae & & & & & & & & & & \\
\hline 31 & Garcinia $\times$ mangostana L. [WS230] & Tree & Manggis & Fruit & 1 & 1 & 1 & 1 & 1 & $\begin{array}{l}\text { C, P, } \\
\text { S, T }\end{array}$ & 13 \\
\hline & & & & Leaves & 1 & 1 & 1 & & & $C, S$ & 4 \\
\hline & Compositae & & & & & & & & & & \\
\hline 32 & Blumea balsamifera (L.) DC. [WS231] & Tree & Sembung & Leaves & & 1 & & 1 & & S & 2 \\
\hline 33 & Cosmos sulphureus Cav. [WS232] & Herb & Padang berman & Leaves & & 1 & & & & C, $\mathrm{P}, \mathrm{T}$ & 13 \\
\hline 34 & Tagetes erecta L. [WS233] & Herb & Gumitir & Flower & 1 & 1 & 1 & 1 & 1 & $\begin{array}{l}\text { C, P, } \\
\text { S, T }\end{array}$ & 20 \\
\hline & Cucurbitaceae & & & & & & & & & & \\
\hline 35 & Benincasa hispida (Thunb.) Cogn. [WS234] & Climber & Blego & Fruit & & 1 & & & & S & 2 \\
\hline 36 & Cucumis sativus L. [WS235] & Climber & Ketimun & Fruit & 1 & 1 & 1 & 1 & 1 & $\mathrm{C}, \mathrm{T}$ & 6 \\
\hline 37 & Cucurbita neno L [WS236] & Climber & Waluh/labu & Leaves & & 1 & & & & P, S & 7 \\
\hline & & & & Fruit & & 1 & & & & P, S & 7 \\
\hline 38 & Momordica charantia I [WS237] & Climber & Pava & Leaves & & 1 & & 1 & & $C$ & 2 \\
\hline & & & & Fruit & & 1 & & 1 & & $S$ & 2 \\
\hline & Dioscoreaceae & & & & & & & & & & \\
\hline 39 & Dioscorea alata L. [WS238] & Climber & $\begin{array}{l}\text { Ubi aung/ubi } \\
\text { liyan }\end{array}$ & Tuber & & 1 & 1 & 1 & & $\begin{array}{l}\text { C, P, } \\
\text { S, T }\end{array}$ & 17 \\
\hline 40 & Dioscorea hispida Dennst. [WS239] & Climber & Gadung & Flower & 1 & 1 & 1 & 1 & 1 & C & 2 \\
\hline & Euphorbiaceae & & & & & & & & & & \\
\hline 41 & Aleurites moluccanus (L.) Willd. [WS240] & Tree & Kemiri/ tingkih & Seeds & 1 & 1 & 1 & 1 & 1 & C, P, S & 9 \\
\hline 42 & Manihot esculenta Crantz [WS241] & Shrub & Sela sawi/ketela & Tuber & 1 & 1 & 1 & 1 & 1 & $C, \mathrm{~T}$ & 5 \\
\hline & Lamiaceae & & & & & & & & & & \\
\hline 43 & Elsholtzia pubescens Benth. [WS242] & Shrub & Junggul & Leaves & & 1 & & & & C & 3 \\
\hline
\end{tabular}


Table 2. Cont.

\begin{tabular}{|c|c|c|c|c|c|c|c|c|c|c|c|}
\hline \multirow[b]{2}{*}{ No. } & \multirow[b]{2}{*}{$\begin{array}{l}\text { Plant families \& species, } \\
\text { [voucher specimen code] }\end{array}$} & \multirow[b]{2}{*}{ Life form } & \multirow[b]{2}{*}{ Vernacular names } & \multirow[b]{2}{*}{$\begin{array}{l}\text { Used } \\
\text { parts }\end{array}$} & \multicolumn{5}{|c|}{ Use in yadnya } & \multirow[b]{2}{*}{ Villages } & \multirow[b]{2}{*}{$\begin{array}{l}\text { Number of } \\
\text { Informants }\end{array}$} \\
\hline & & & & & $\begin{array}{c}\text { Dewa } \\
\text { yadnya }\end{array}$ & $\begin{array}{c}\text { Pitra } \\
\text { yadnya }\end{array}$ & $\begin{array}{l}\text { Manusa } \\
\text { yadnya }\end{array}$ & $\begin{array}{c}\text { Bhuta } \\
\text { yadnya }\end{array}$ & $\begin{array}{c}\text { Rsi } \\
\text { yadnya }\end{array}$ & & \\
\hline \multirow[t]{2}{*}{44} & Plectranthus scutellarioides (L.) R.Br. [WS243] & Shrub & Reng-reng & Leaves & 1 & 1 & & 1 & & $\mathrm{P}$ & 2 \\
\hline & Leguminosae & & & & & & & & & & \\
\hline 45 & Arachis hypogaea L. [WS244] & Herb & Kacang tanah & Seeds & 1 & 1 & 1 & 1 & 1 & C & 2 \\
\hline 46 & Caesalpinia pulcherrima (L.) Sw. [WS245] & Shrub & Kemerakan & Flower & & & & 1 & & $\mathrm{~T}$ & 3 \\
\hline 47 & Cajanus cajan (L.) Millsp. [WS246] & Shrub & Undis & Seeds & 1 & 1 & 1 & 1 & 1 & $\begin{array}{l}\text { C, P, } \\
\text { S, T }\end{array}$ & 7 \\
\hline 48 & Canavalia gladiata (Jacq.) DC. [WS247] & Climber & Juleh & Leaves & & 1 & & & & P, S & 3 \\
\hline 49 & Clitoria ternatea L. [WS248] & Climber & Teleng & Flower & & 1 & & & & $C$ & 2 \\
\hline 50 & Entada phaseoloides (L.) Merr. [WS249] & Climber & Cikal & Fruit & 1 & & & 1 & & $\mathrm{P}$ & 1 \\
\hline 51 & Erythrina crista-galli L. [WS250] & Tree & Canging & Leaves & & 1 & & & & S & 2 \\
\hline \multirow[t]{2}{*}{52} & \multirow{2}{*}{ Erythrina subumbrans (Hassk.) Merr. [WS251] } & \multirow{2}{*}{ Tree } & \multirow{2}{*}{ Dadap tis } & Leaves & 1 & 1 & 1 & 1 & 1 & $\begin{array}{l}\text { C, P, } \\
\mathrm{S}, \mathrm{T}\end{array}$ & 17 \\
\hline & & & & Wood & 1 & 1 & 1 & 1 & 1 & C, S, T & 8 \\
\hline 53 & Lablab purpureus (L.) Sweet [WS252] & Climber & Komak & Seeds & 1 & 1 & 1 & 1 & 1 & C, S, T & 13 \\
\hline 54 & Psophocarpus tetragonolobus (L.) DC. [WS253] & Climber & Botor & Seeds & & 1 & 1 & 1 & & $\begin{array}{l}\mathrm{C}, \mathrm{P} \\
\mathrm{S}, \mathrm{T}\end{array}$ & 11 \\
\hline 55 & Pueraria phaseoloides (Roxb.) Benth. [WS254] & Climber & Ucu & Seeds & 1 & 1 & 1 & 1 & 1 & $\mathrm{P}, \mathrm{S}, \mathrm{T}$ & 7 \\
\hline \multirow[t]{2}{*}{56} & Vigna unguiculata (L.) Walp. [WS255] & Climber & Kacang panjang & Fruit & 1 & 1 & 1 & 1 & 1 & C & 2 \\
\hline & $\begin{array}{l}\text { Lygodiaceae } \\
\end{array}$ & & & & & & & & & & \\
\hline \multirow[t]{2}{*}{57} & Lygodium circinatum (Burm. f.) Sw. [WS256] & Climber & Paku ata & Leaves & & 1 & 1 & 1 & & $\mathrm{P}$ & 3 \\
\hline & $\begin{array}{l}\text { Lythraceae } \\
\end{array}$ & & & & & & & & & & \\
\hline 58 & Punica granatum L. [WS257] & Shrub & Delima & Fruit & 1 & 1 & 1 & 1 & 1 & $C$ & 3 \\
\hline \multirow[t]{2}{*}{59} & Michelia alba DC. [WS258] & Tree & Cempaka & Flower & 1 & & 1 & & 1 & $\begin{array}{l}\text { C, P, } \\
\text { S, T }\end{array}$ & 13 \\
\hline & Malvaceae & & & & & & & & & & \\
\hline \multirow{2}{*}{60} & \multirow{2}{*}{ Durio zibethinus L. [WS259] } & \multirow{2}{*}{ Tree } & \multirow{2}{*}{ Durian } & Fruit & 1 & 1 & 1 & 1 & 1 & $C, S$ & 7 \\
\hline & & & & Leaves & & 1 & & & & $C$ & 2 \\
\hline 61 & Hibiscus rosa-sinensis L. [WS260] & Shrub & Pucuk bang & Flower & & 1 & & & & C, S, T & 10 \\
\hline & & & & Leaves & & 1 & 1 & & & $\mathrm{P}$ & 1 \\
\hline 62 & Melochia umbellata (Houtt.) Stapf [WS261] & Tree & Bentenu & Bark & & 1 & & & & $\mathrm{P}$ & 1 \\
\hline & & & & Wood & & 1 & 1 & & & C & 3 \\
\hline & Marantaceae & & & & & & & & & & \\
\hline 63 & Maranta arundinacea L. [WS262] & Shrub & Celengidi & Leaves & & 1 & & & & $\mathrm{P}$ & 1 \\
\hline & Meliaceae & & & & & & & & & & \\
\hline 64 & Lansium parasiticum (Osbeck) K.C.Sahni \& & Tree & Ceroring & Fruit & 1 & 1 & 1 & 1 & 1 & $\begin{array}{l}\text { C, P, } \\
\text { S, T }\end{array}$ & 13 \\
\hline & Bennet [WS263] & & & Bark & & & 1 & & & C & 2 \\
\hline 65 & $\begin{array}{c}\text { Dysoxylum parasiticum (Osbeck) Kosterm. } \\
\text { [WS264] }\end{array}$ & Tree & Majagau & Wood & 1 & 1 & 1 & 1 & 1 & C, P, S & 13 \\
\hline 66 & Sandoricum koetjape (Burm.f.) Merr. [WS265] & Tree & Sentul & Fruit & & 1 & & 1 & & $\mathrm{P}, \mathrm{S}, \mathrm{T}$ & 8 \\
\hline & Moraceae & & & & & & & & & & \\
\hline 67 & Artocarpus heterophyllus Lam. [WS266] & Tree & Nangka & Fruit & 1 & 1 & 1 & 1 & 1 & C & 3 \\
\hline 68 & Ficus benjamina L. [WS267] & Tree & Bingin & Leaves & & 1 & & & & C, P, S & 10 \\
\hline & Moringaceae & & & & & & & & & & \\
\hline 69 & Moringa oleifera Lam. [WS268] & Tree & Kelor & Leaves & & 1 & & & & C & 2 \\
\hline & Musaceae & & & & & & & & & & \\
\hline 70 & Musa $\times$ paradisiaca L. [WS269] & Herb & $\begin{array}{c}\text { Pisang/biu/ } \\
\text { dak/biu susu/ } \\
\text { biu mas/biu } \\
\text { raja/biu kayu/ } \\
\text { biu bunga/biu } \\
\text { gancah/biu } \\
\text { tembaga }\end{array}$ & Leaves & 1 & 1 & 1 & 1 & 1 & $\begin{array}{l}C, P, \\
S, T\end{array}$ & 20 \\
\hline & Myrtaceae & & & & & & & & & & \\
\hline 71 & Psidium guajava L. [WS270] & Tree & Sotong & Fruit & 1 & 1 & 1 & 1 & 1 & $\begin{array}{l}\text { C, P, } \\
\text { S, T }\end{array}$ & 15 \\
\hline 72 & $\begin{array}{c}\text { Syzygium polycephalum (Miq.) Merr. \& } \\
\text { L.M.Perry [WS271] }\end{array}$ & Tree & Kaliasem & Fruit & & 1 & 1 & & & $\mathrm{P}$ & 2 \\
\hline
\end{tabular}


Table 2. Cont.

\begin{tabular}{|c|c|c|c|c|c|c|c|c|c|c|c|}
\hline \multirow[b]{2}{*}{ No. } & \multirow[b]{2}{*}{$\begin{array}{l}\text { Plant families \& species, } \\
\text { [voucher specimen code] }\end{array}$} & \multirow[b]{2}{*}{ Life form } & \multirow[b]{2}{*}{ Vernacular names } & \multirow[b]{2}{*}{$\begin{array}{l}\text { Used } \\
\text { parts }\end{array}$} & \multicolumn{5}{|c|}{ Use in yadnya } & \multirow[b]{2}{*}{ Villages } & \multirow[b]{2}{*}{$\begin{array}{l}\text { Number of } \\
\text { Informants }\end{array}$} \\
\hline & & & & & $\begin{array}{c}\text { Dewa } \\
\text { yadnya }\end{array}$ & $\begin{array}{c}\text { Pitra } \\
\text { yadnya }\end{array}$ & $\begin{array}{l}\text { Manusa } \\
\text { yadnya }\end{array}$ & $\begin{array}{l}\text { Bhuta } \\
\text { yadnya }\end{array}$ & $\begin{array}{c}\text { Rsi } \\
\text { yadnya }\end{array}$ & & \\
\hline \multirow{3}{*}{73} & Nephrolepidaceae & & & & & & & & & & \\
\hline & Nephrolepis cordifolia (L.) C. Presl [WS272] & Herb & $\begin{array}{l}\text { Paku pipid/ } \\
\text { paku lipan }\end{array}$ & Leaves & 1 & 1 & & 1 & & C & 5 \\
\hline & Nymphaeaceae & & & & & & & & & & \\
\hline 74 & Nymphaea lotus L. [WS273] & Herb & Tunjung & Flower & 1 & 1 & 1 & 1 & 1 & C, S, T & 13 \\
\hline \multirow[t]{2}{*}{75} & $\begin{array}{l}\text { Pandanaceae } \\
\text { Pandanus tectorius Parkinson ex Du Roi } \\
\text { [WS274] }\end{array}$ & Shrub & Pandan/pudak & Leaves & 1 & 1 & 1 & 1 & 1 & C, P, S & 14 \\
\hline & Phyllanthaceae & & & & & & & & & & \\
\hline 76 & Antidesma bunius (L.) Spreng. [WS275] & Tree & Buni & Fruit & 1 & 1 & 1 & & & $\mathrm{P}$ & 2 \\
\hline \multirow{2}{*}{77} & \multirow{2}{*}{$\begin{array}{l}\text { Baccaurea racemosa (Reinw. ex Blume) Müll.Arg. } \\
\text { [WS276] }\end{array}$} & \multirow{2}{*}{ Tree } & \multirow{2}{*}{ Kepundung } & Fruit & 1 & 1 & 1 & 1 & 1 & $\begin{array}{l}\text { C, P, } \\
\text { S, T }\end{array}$ & 14 \\
\hline & & & & Leaves & & 1 & & & & C & 2 \\
\hline \multirow[t]{2}{*}{78} & \multirow{2}{*}{ Phyllanthus emblica L [WS277] } & \multirow{2}{*}{ Tree } & \multirow{2}{*}{ Kalimoko } & Fruit & & 1 & & 1 & & C & 5 \\
\hline & & & & Leaves & & 1 & & & & $C$ & 2 \\
\hline 79 & $\begin{array}{c}\text { Phyllanthus buxifolius (Blume) Müll.Arg. } \\
\text { [WS278] }\end{array}$ & Shrub & Sisih & Wood & & 1 & & 1 & & $\begin{array}{l}\text { C, P, } \\
\text { S, T }\end{array}$ & 15 \\
\hline \multirow[t]{2}{*}{80} & Phyllanthus niruri L. [WS279] & Shrub & Menirang & Leaves & & 1 & & & & $\mathrm{P}$ & 4 \\
\hline & Piperaceae & & & & & & & & & & \\
\hline \multirow[t]{2}{*}{81} & Piper betle L. [WS280] & Climber & Sirih & Leaves & 1 & 1 & 1 & 1 & 1 & $\begin{array}{l}\text { C, P, } \\
\text { S, T }\end{array}$ & 20 \\
\hline & Poaceae & & & & & & & & & & \\
\hline 82 & Bambusa vulgaris Schrad. [WS281] & Tree & $\begin{array}{l}\text { Tiing ampel } \\
\text { gading }\end{array}$ & Culm & & 1 & & & & C, P, S & 8 \\
\hline 83 & Coix lacryma-jobi L. [WS282] & Herb & Jali-jali & Seeds & & 1 & & 1 & & $\begin{array}{l}C, P, \\
S, T\end{array}$ & 16 \\
\hline 84 & Cynodon dactylon (L.) Pers. [WS283] & Herb & Padang lepas & Leaves & & 1 & & & & C, P, & 13 \\
\hline 85 & Dendrocalamus asper (Schult.) Backer [WS284] & Tree & Tiing jelepung & Culm & 1 & 1 & 1 & 1 & 1 & $C, P$ & 4 \\
\hline 86 & Eleusine coracana (L.) Gaertn. [WS285] & Herb & Godem & Seeds & & 1 & & 1 & & $\begin{array}{l}\text { C, P, } \\
\text { S, T }\end{array}$ & 16 \\
\hline 87 & Eleusine indica (L.) Gaertn. [WS286] & Herb & $\begin{array}{c}\text { Padang } \\
\text { belulang }\end{array}$ & Leaves & & 1 & & & & $\mathrm{P}$ & 2 \\
\hline 88 & Gigantochloa apus (Schult.) Kurz [WS287] & Tree & Tiing tali & Culm & 1 & 1 & 1 & 1 & 1 & C, S, T & 8 \\
\hline 89 & Gigantochloa baliana Widjaja \& Astuti [WS288] & Tree & Tiing bali & Culm & & 1 & & & & $\begin{array}{l}\text { C, P, } \\
\text { S, T }\end{array}$ & 15 \\
\hline 90 & Imperata cylindrica (L.) Raeusch. [WS289] & Herb & Lalang & Leaves & 1 & 1 & 1 & 1 & 1 & C, S & 12 \\
\hline 91 & Oryza sativa L. [WS290] & Herb & $\begin{array}{l}\text { Padi/padi gaga/ } \\
\text { ketan/ injin }\end{array}$ & Seeds & 1 & 1 & 1 & 1 & 1 & C, P, & 20 \\
\hline 92 & Panicum miliaceum L. [WS291] & Herb & Jawe & Seeds & 1 & 1 & & & & $\begin{array}{l}\text { C, P, } \\
S, T\end{array}$ & 16 \\
\hline 93 & Saccharum officinarum L. [WS292] & Herb & Tebu cemeng & Stem & 1 & 1 & 1 & 1 & 1 & $\begin{array}{l}\text { C, P, } \\
\text { S, T }\end{array}$ & 20 \\
\hline 94 & $\begin{array}{c}\text { Schizostachyum brachycladum (Kurz) Kurz } \\
\text { [WS293] }\end{array}$ & Tree & $\begin{array}{l}\text { Tiing buluh } \\
\text { gading/ } \\
\text { tamblang }\end{array}$ & Culm & & 1 & & 1 & & C & 5 \\
\hline 95 & Schizostachyum lima (Blanco) Merr. [WS294] & Tree & Tiing buluh & Culm & & 1 & & 1 & & $\mathrm{P}, \mathrm{T}$ & 4 \\
\hline & & & & Leaves & & 1 & & 1 & & $\mathrm{P}$ & 1 \\
\hline 96 & Sorghum bicolor (L.) Moench [WS295] & Herb & Jagung kedu & Seeds & & 1 & & & & $\begin{array}{l}\text { C, P, } \\
\text { S, T }\end{array}$ & 15 \\
\hline & Pteridaceae & & & & & & & & & & \\
\hline 97 & Pityrogramma calomelanos (L.) Link [WS296] & Herb & Paku sudamala & Leaves & & 1 & & & & $\mathrm{P}, \mathrm{T}$ & 4 \\
\hline & Rosaceae & & & & & & & & & & \\
\hline 98 & Rubus buergeri Miq. [WS297] & Shrub & $\begin{array}{l}\text { Gunggung } \\
\text { bukit }\end{array}$ & Leaves & & 1 & & & & C, P, S & 9 \\
\hline 99 & Rubus rosifolius Sm. [WS298] & Shrub & $\begin{array}{l}\text { Gunggung bali/ } \\
\text { lengis }\end{array}$ & Leaves & & 1 & & 1 & & C, P, S & 11 \\
\hline & Rubiaceae & & & & & & & & & & \\
\hline & Gardenia iasminoides JEllis [WS299] & Shrub & & Flower & 1 & 1 & 1 & 1 & 1 & P, S & 6 \\
\hline 100 & Gardenia jasminoides J.Ellis [WS299] & Shrub & Jempiring & Leaves & 1 & 1 & & & & $\mathrm{P}$ & 1 \\
\hline 101 & Morinda citrifolia L. [WS300] & Tree & Tibah & Fruit & 1 & 1 & 1 & 1 & 1 & $\mathrm{P}$ & 1 \\
\hline 102 & $\begin{array}{c}\text { Neonauclea calycina (Bartl. ex DC.) Merr. } \\
\text { [WS301 }\end{array}$ & Tree & Daun bengkel & Leaves & & 1 & & & & $\mathrm{~T}$ & 2 \\
\hline
\end{tabular}


Table 2. Cont.

\begin{tabular}{|c|c|c|c|c|c|c|c|c|c|c|c|}
\hline \multirow[b]{2}{*}{ No. } & \multirow{2}{*}{$\begin{array}{l}\text { Plant families \& species, } \\
\text { [voucher specimen code] }\end{array}$} & \multirow[b]{2}{*}{ Life form } & \multirow[b]{2}{*}{ Vernacular names } & \multirow[b]{2}{*}{$\begin{array}{l}\text { Used } \\
\text { parts }\end{array}$} & \multicolumn{5}{|c|}{ Use in yadnya } & \multirow[b]{2}{*}{ Villages } & \multirow[b]{2}{*}{$\begin{array}{l}\text { Number of } \\
\text { Informants }\end{array}$} \\
\hline & & & & & \begin{tabular}{|c|} 
Dewa \\
yadnya
\end{tabular} & $\begin{array}{c}\text { Pitra } \\
\text { yadnya }\end{array}$ & $\begin{array}{l}\text { Manusa } \\
\text { yadnya }\end{array}$ & $\begin{array}{c}\text { Bhuta } \\
\text { yadnya }\end{array}$ & $\begin{array}{c}\text { Rsi } \\
\text { yadnya }\end{array}$ & & \\
\hline & Rutaceae & & & & & & & & & & \\
\hline 103 & Citrus $\times$ aurantium L. [WS302] & Tree & Semaga & Fruit & 1 & 1 & 1 & 1 & 1 & $\mathrm{C}, \mathrm{P}$ & 2 \\
\hline 104 & Citrus maxima (Burm.) Merr. [WS303] & Tree & Jeruk & Fruit & 1 & 1 & 1 & 1 & 1 & C, $\mathrm{P}, \mathrm{T}$ & 6 \\
\hline \multirow[t]{2}{*}{105} & Murraya koenigii (L.) Spreng. [WS304] & Tree & Pupug & Wood & & 1 & & & & C, P, S & 9 \\
\hline & Santalaceae & & & & & & & & & & \\
\hline \multirow[t]{2}{*}{106} & Santalum album L. [WS305] & Tree & Cendana & Wood & 1 & 1 & 1 & 1 & 1 & C, P, S & 12 \\
\hline & Sapindaceae & & & & & & & & & & \\
\hline \multirow{3}{*}{107} & Nonhelium lannaceum I [WTS306] & Tree & Buluan/ & Fruit & 1 & 1 & 1 & 1 & 1 & C, S & 3 \\
\hline & Nephelium lappaceum L. [WS306] & Tree & rambutan & Leaves & & 1 & & & & $C$ & 2 \\
\hline & Sapotaceae & & & & & & & & & & \\
\hline \multirow[t]{2}{*}{108} & Manilkara zapota (L.) P.Royen [WS307] & Tree & Sawo & Fruit & 1 & 1 & 1 & 1 & 1 & C, $\mathrm{P}$ & 4 \\
\hline & Selaginellaceae & & & & & & & & & & \\
\hline \multirow[t]{2}{*}{109} & Selaginella delicatula (Desv. Ex Poir.) [WS308] & Herb & Bekenying & Leaves & 1 & 1 & & & & $\mathrm{P}$ & 2 \\
\hline & Solanaceae & & & & & & & & & & \\
\hline 110 & Capsicum annuum L [WS309] & Shrub & Cabai/tabia & Fruit & 1 & 1 & 1 & 1 & 1 & $\mathrm{C}, \mathrm{S}, \mathrm{T}$ & 5 \\
\hline \multirow[t]{2}{*}{111} & Solanum melongena L. [WS310] & Shrub & $\begin{array}{l}\text { Tuwung/ } \\
\text { terung }\end{array}$ & Fruit & & 1 & & & & $C, \mathrm{~T}$ & 5 \\
\hline & Styracaceae & & & & & & & & & & \\
\hline \multirow[t]{2}{*}{112} & Styrax benzoin Dryand. [WS311] & Tree & Menyan & Wood & 1 & 1 & 1 & 1 & 1 & C, P, S & 13 \\
\hline & Urticaceae & & & & & & & & & & \\
\hline 113 & Boehmeria nivea (L.) Gaudich. [WS312] & Shrub & Bagu & Leaves & & 1 & & & & $\mathrm{P}$ & 1 \\
\hline \multirow{2}{*}{114} & \multirow{2}{*}{ Dendrocnide stimulans (L.f.) Chew [WS313] } & \multirow{2}{*}{ Tree } & \multirow{2}{*}{ Lateng } & Leaves & & 1 & & & & C, P, S & 8 \\
\hline & & & & Wood & & 1 & & & & $\mathrm{P}$ & 1 \\
\hline \multirow[t]{2}{*}{115} & Leucosyke capitellata Wedd. [WS314] & Tree & Patih kalah & Leaves & & 1 & & & & $C, P$ & 7 \\
\hline & Vitaceae & & & & & & & & & & \\
\hline 116 & Cissus javana DC. [WS315] & Climber & Dinding ai & $\begin{array}{l}\text { Whole } \\
\text { part }\end{array}$ & 1 & & & & & C, P, S & 11 \\
\hline \multirow[t]{2}{*}{117} & Leea angulata Korth. ex Miq. [WS316] & Shrub & Kelawasan & Leaves & & 1 & & & & $\mathrm{P}$ & 2 \\
\hline & Zingiberaceae & & & & & & & & & & \\
\hline 118 & Alpinia galanga (L.) Willd. [WS317] & Herb & Isen/ lengkuas & Tuber & 1 & 1 & 1 & 1 & 1 & $\mathrm{P}, \mathrm{T}$ & 4 \\
\hline 119 & Amomum maximum Roxb. [WS318] & Herb & Kase & Fruit & & 1 & & & & $\mathrm{P}$ & 3 \\
\hline 120 & Curcuma viridiflora Roxb. [WS319] & Herb & Kunir/kunyit & Tuber & 1 & 1 & 1 & 1 & 1 & $C, P$ & 7 \\
\hline \multirow{2}{*}{121} & \multirow{2}{*}{ Curcuma zedoaria (Christm.) Roscoe [WS320] } & \multirow{2}{*}{ Herb } & Kenanogean & Leaves & & 1 & & & & $C$ & 3 \\
\hline & & & & Tuber & & 1 & & & & $S$ & 2 \\
\hline 122 & Etlingera elatior (Jack) R.M.Sm. [WS321] & Herb & Kecicang & Flower & 1 & & & & & C, S & 7 \\
\hline 123 & Kaempferia rotunda L. [WS322] & Herb & Cekuh/kencur & Tuber & 1 & 1 & 1 & 1 & 1 & $\mathrm{C}, \mathrm{T}$ & 4 \\
\hline 124 & $\begin{array}{c}\text { Zingiber montanum (J.Koenig) Link ex A.Dietr. } \\
\text { [WS323] }\end{array}$ & Herb & Gamongan & Tuber & & 1 & & 1 & & $\mathrm{P}$ & 1 \\
\hline 125 & Zingiber officinale Roscoe [WS324] & Herb & Jahe & Tuber & 1 & 1 & 1 & 1 & 1 & $\mathrm{C}, \mathrm{P}, \mathrm{T}$ & 9 \\
\hline
\end{tabular}

plants are not accidentally used. Each plant can then express a precise meaning related to ceremonies and offerings in which it occurs and provides a specific word of an elaborate vocabulary of symbols to show devotion to gods (Barth 1993).

Plant species present in offerings are often native (Girmansyah et al. 2013) or easily reachable by the Balinese because they are cultivated in home gardens, otherwise commonly sold in traditional markets (Sujarwo et al. 2018). Moreover, alien species (e.g., Celosia spicata, Eleusine indica, Imperata cylindrica, Phyllanthus niruri) are wellknown and frequently used by the Balinese. Among the non-indigenous species, it is noteworthy to mention the coincidence of the first appearance in Indonesia of species of the Indian region (e.g., Cajanus cajan, Cucumis sativus,
Momordica charantia, Solanum melongena, Tabernaemontana divaricata) with the introduction of religious and cultural Indian influences in the eighth century (Rao 2001). Other plants have been incorporated later into the offering. For instance, the Dutch were responsible for the introduction of plants native to Central and South America (e.g., Arachis hypogea, Capsicum annuum, Carica papaya, Manihot esculenta, Plumeria alba, Psidium guajava, Tagetes erecta) during the sixteenth century (Simmonds 1976). Also, the selection of species (Silva et al. 2018) seems to follow criteria (e.g., aesthetic such as colors, shapes, smell; apotropaic; curative; food; function) based on the knowledge on the local flora, occurrence and abundance in the natural environment, common presence in home gardens and old traditional uses. 
There are some studies on the Traditional Ecological Knowledge of Bali about its specific aspects, its structure, and the cultural erosion that affects it (Sujarwo et al. 2014; 2016; Caneva et al. 2017; Sujarwo \& Lestari 2018). The local knowledge about the plants used in the Panca yadnya seems based on the Traditional Ecological Knowledge about the general ethnoflora of Bali as people use wellknown ethnobotanical species. For this reason, it should be interpreted as a particular subset of general TEK and could also show the same risk of cultural erosion due to global transformation processes (see Sujarwo et al. 2014).

Pools of plants in yadnyas and floristic similarities between yadnyas

Panca yadnya is different forms of worship with specific rituals and offerings. Each ceremony belongs to a single yadnya, but elements of ceremonies of other yadnyas can actually be included (Putra 1988). The type of yadnya does not strictly constrain the ceremony as it is mostly focused on the rituals and offerings. As such, it is not surprisingly that a large pool of plants is shared in all yadnyas. On the other hand, there are several pools that are specific to a single yadnya according to its types of rituals. For instance, the offerings in Pitra and Butha yadnyas, which are quite particular in Balinese Hinduism, show a low similarity to other offerings and are highlighted by exclusive and big contingents of plant parts. On the other hand, the offerings in Dewa yadnya are characterized by a small specific pool and several species shared by other yadnyas. Balinese Hindus must perform several life-cycle rituals (Manusa yadnya) during their lifetime (Ariati 2006). In this case, the offering composition also shows connections to other yadnyas with some pools of shared plants, but to a lesser extent. Few ceremonies of ordination belong to the Rsi yadnya. There is no exclusive pool of plants for such yadnya, but contingents of plants from other yadnyas can be used for its offerings.

Managing the religious relationships between men and god seems to be the most important factor in determining the plant compositions in the yadnyas. In these compositions, it is noteworthy pervasive influence of worship to gods and animistic forms of deities in Balinese Hinduism. Rsi yadnya appears excluded from relationships among Pitra, Butha, Manusa, and Dewa. Only one species, i.e., Cananga odorata, whose flowers symbolize the preserver (Vishnu) is common in all yadnyas. Parts of some species are also selectively used in distinct kinds of ceremonies and offerings. The most important one is Arenga pinnata, already identified in a previous study (Sujarwo \& Lestari 2018), as a Cultural Keystone Species of Bali (see Garibaldi \& Turner 2004).

In general, it seems possible to infer many typical religious relationships (Bhuta, Dewa, Manusa, Pitra, and $R s i$ in Panca yadnya from the offering compositions based on pools of plant parts. In addition, different species pools could better specify and clarify spiritual and religious ecosystem services (Hernández-Morcillo et al. 2013) in the Balinese cultural context. The structure of knowledge about the ethnoflora of offerings shows two main parts: the common or nuclear, and the specific, restricted to only one yadnya. In a previous study about another aspect of TEK in Bali (Caneva et al. 2017), the same structure was detected, and parts of knowledge were described as core and satellite groups. This fact suggests that the knowledge about the ethnoflora of offerings, as a specific subset of the general TEK, could have the same pattern.

\section{Orthopraxy of religious customs and ethnoflora of offerings in Panca yadnya}

The set of yadnyas, Panca yadnya, represents a model of orthopraxy in Balinese Hinduism and could help to better detect and describe general symbolic features of different aspects of religion in Bali (Hornbacher 2011). The current dataset about plants and their parts used in the religious ceremonies of the Panca yadnya is based on knowledge of religious leaders and local people involved in the making of offerings and provides a vision on the orthopraxy of Balinese Hinduism related to the utilization of different plant materials. Sometimes, in Bali, traditional practices may be the only way to find and assess the actual relevance of plants and their particular relationships to local religion. Orthopraxy could then be used as a powerful tool to describe general symbolic meanings of species by their link to different kinds of ceremonies, as this study suggests. Moreover, the considerable importance of orthopraxy in Balinese Hinduism ensures a very conservative religious system, which was occurring since $15^{\text {th }}$ century, and a permanent and stable related corpus of TEK, properly safeguarding the knowledge about the ethnoflora of offerings from cultural erosion and transformation processes, as already suggested by Eiseman (1990).

\section{Conclusion}

Offerings in Balinese Hinduism are material objects to offer for religious purposes and they also represent one of the most important manners to interpret and practice religion in everyday life. Stating their relevance in the religious culture of Bali, this study performed the first attempt to define an ethnoflora of offerings. This study also constitutes an essential step towards a complete ethnobotanical description of Balinese Hinduism, giving it a conceptual framework to evaluate cultural ecosystem services provided by the plants used in religious ceremonies.

Balinese Hinduism, embodying particular animistic aspects of the indigenous religion, along with its ceremonies and offering compositions, can express Balinese cosmology better than doctrinal texts. The practices of religious rituals seem fundamental to verify the general religious meaning of plants and their great importance for the Balinese to maintain the integrity of TEK related to ethnoflora of 
offerings, avoiding general and global phenomena of loss, cultural erosion and transformation.

\section{Acknowledgements}

We would like to express our gratitude to the informants who took part in our study and for sharing their knowledge, hospitality, and assistance. We also express our appreciation to I Nyoman Peneng and I Nyoman Sudiatna for their assistance during fieldwork, I Gede Wawan Setiadi for preparing the pictures of Panca yadnya and Arenga pinnata. This study was funded by the Indonesian Institute of Sciences (LIPI) through the Bali Botanical Gardens for the field investigations [Letter of Assignment No.B-1288/IPH.7/KP/XII/2017].

\section{References}

Acri A. 2011. A new perspective for 'Balinese Hinduism' in the light of the premodern religious discourse: a textual-historical approach. In: Picard M, Madinier R. (eds.) The politics of religion in Indonesia: syncretism, orthodoxy, and religious contention in Java and Bali. New York, Routledge. p. 142-166.

Alexiades MN, Sheldon JW. 1996. Selected guidelines for ethnobotanical research: a field manual. New York, The New York Botanical Garden Press.

Ariati NWP. 2006. Journey of the Goddess Durga: India Java and Bali. New Delhi, Aditya Prakashan.

Bakan MB. 2011. Preventive care for the dead: music, community, and the protection of souls in Balinese cremation ceremonies. In: Koen BD. (eds.) The oxford handbook of medical ethnomusicology. Oxford, Oxford University Press. p. 1-19.

Barth F. 1993. Balinese worlds. Chicago, University of Chicago Press.

Belo J. 1960. Trance in Bali. New York, Columbia University Press.

Bernard HR. 2002. Research methods in anthropology: qualitative and quantitative approaches. Walnut Creek California, Altamira Press.

BPS. 2017. Bali dalam angka 2016. Denpasar, Badan Pusat Statistik Press.

Caneva G, Traversetti L, Sujarwo W, Zuccarello V. 2017. Sharing ethnobotanical knowledge in traditional villages: evidence of food and nutraceutical "core groups" in Bali, Indonesia. Economic Botany 71: 303-313.

Covarrubias M. 1937. Island of Bali. Singapore, Periplus.

Cush D, Robinson C, York M. 2008. Encyclopedia of Hinduism. London, Routledge.

Dibia IW, Ballinger R. 2011. Balinese dance, drama \& music: a guide to the performing arts of Bali. Singapore, Tutle Publishing.

Eiseman Jr FB. 1990. Bali: sekala and niskala: essays on religion, ritual, and art. Singapore, Tutle Publishing.

Garibaldi A, Turner N. 2004. Cultural keystone species: implication for ecological conservation and restoration. Ecology and Society 9(3): 1. https://www.ecologyandsociety.org/vol9/iss3/art1/

Geertz C. 1973. The interpretation of cultures: selected essays. New York, Basic Books Inc.

Geertz C. 1980. Negara: the theatre state in $19^{\text {th }}$ century Bali. Princeton, Princeton University Press.

Geertz H, Geertz C. 1975. Kinship in Bali. Chicago, University of Chicago Press.

Girmansyah D, Santika Y, Retnowati A, et al. 2013. Flora of Bali: an annotated checklist. Jakarta, Yayasan Pustaka Obor Indonesia.

Hernández-Morcillo M, Plieninger T, Bieling C. 2013. An empirical review of cultural ecosystem service indicators. Ecological Indicators 29: 434-444.

Hobart A, Ramseyer U, Leeman A. 2001. The people of Bali. Hoboken, Wiley-Blackwell.

Hockings P. 1993. Encyclopedia of world cultures Vol. 4: East and Southeast Asia. New York, G.K. Hall \& Company.

Hornbacher A. 2011. The withdrawal of the Gods: Remarks on ritualtrancepossession and its decline in Bali. In: Picard M, Madinier R. (eds.) The politics of religion in Indonesia: syncretism, orthodoxy, and religious contention in Java and Bali. New York, Routledge. p. 167-191.

ISE - International Society of Ethnobiology. 2006. International society of ethnobiology code of ethics (with 2008 additions). Florida, Department of Anthropology, University of Florida. https://www.ethnobiology.net/ ethics.php. 12 Jun. 2019

Jaccard P. 1901. Étude comparative de la distribution florale dans une portion des Alpes et des jura. Bulletin de la Société Vaudoise des Sciences Naturelles 37: 547-579.

Jansen E. 1993. The book of Hindu imaginary: gods, manifestation and their meaning. Havelte, Binkey Kok Publications BV.

Jones CA, Ryan JR. 2007. Encyclopedia of hinduism. New York, Infobase Publishing.

Krishna N. 2017. Hinduism and nature. New Delhi, Penguin India.

Loehr NA. 2017. Combinatorics. 2nd. edn. Boca Raton FL, CRC Press.

Martin GJ. 2004. Ethnobotany: a methods manual. London, Earthscan.

Menendez-Baceta G, Aceituno-Mata L, Tardío J, Reyes-García V, Pardode-Santayana M. 2012. Wild edible plants traditionally gathered in Gorbeialdea (Biscay, Basque Country). Genetic Resources and Crop Evolution 59: 1329-1347.

Monier-Williams M, Leuman E, Cappeller C. 1992. A Sanskrit-English dictionary. Oxford, Oxford University Press.

Picard M. 2011. From agama Hindu Bali to agama Hindu and back: toward a relocalization of the Balinese religion? In: Picard M, Madinier R. (eds.) The politics of religion in Indonesia: syncretism, orthodoxy, and religious contention in Java and Bali. New York, Routledge. p. 117-141.

Putra IGA. 1988. Panca yadnya. Jakarta, Yayasan Dharma Sarathi.

Rao KP. 2001. Early trade and contacts between South India and Southeast Asia (300 B.C. - A.D. 200). East and West 51: 385-394.

Reuter T. 2012. Religion in the age of globalization: emerging trends, Indonesia example. In: Tomlinson M, Manderson L, Smith W. (eds.) Flows of faith: religious reach and community in Asia and the Pacific. Dordrecht, Springer Science+Business Media. p. 13-33.

Rosenthal JP. 2006. Politics, culture, and governance in the development of prior informed consent in indigenous communities. Current Anthropology 47: 119-142.

Sharma A. 2003. The study of Hinduism. Columbia, University of South Carolina Press.

Silva TC, Silva JM, Ramos MA. 2018. What factors guide the selection of medicinal plants in a local pharmacopoeia? a case study in a rural community from a historically transformed Atlantic forest landscape. Evidence-Based Complementary and Alternative Medicine 2018: 1-10.

Simmonds NW. 1976. Evolution of crop plants. Essex, Longman Scientific \& Technical.

Sujarwo W, Arinasa IBK, Caneva G, Guarrera PM. 2016. Traditional knowledge of wild and semi-wild edible plants used in Bali (Indonesia) to maintain biological and cultural diversity. Plant Biosystems 150: 971-976.

Sujarwo W, Arinasa IBK, Salomone F, Caneva G, Fattorini S. 2014. Cultural erosion of Balinese indigenous knowledge of food and nutraceutical plants. Economic Botany 68: 426-437.

Sujarwo W, Caneva G. 2015. Ethnobotanical study of cultivated plants in home gardens of traditional villages in Bali (Indonesia). Human Ecology 43: 769-778.

Sujarwo W, Keim AP, Savo V, Guarrera PM, Caneva G. 2015. Ethnobotanical study of Loloh: traditional herbal drinks from Bali (Indonesia). Journal of Ethnopharmacology 169: 34-48.

Sujarwo W, Lestari SG. 2018. Studi etnobotani tumbuhan obat dan upacara adat Hindu di Bali. Buletin Kebun Raya 21: 117-139.

Sujarwo W, Lugrayasa IN, Kuswantoro F. 2018. Studi etnobotani tiga pasar tradisional di Kabupaten Tabanan Bali. Berita Biologi 17: 283-297.

Takhtajan A. 1986. The floristic region of the world. California, University of California Press.

The Plantlist. 2018. The plantlist database. Royal Botanic Gardens, Kew and Missouri Botanical Garden. http://www.theplantlist.org. 18 Dec. 18.

Wiener MJ. 1995. Visible and invisible realms: Power, magic, and colonial conquest in Bali. Chicago, University of Chicago Press.

Yamashita S. 2003. Bali and Beyond: Explorations in the Anthropology of Tourism (Asian Anthropologies). New York, Berghahn Books. 